\title{
An Open Data Approach to Mapping Urban Drainage Infrastructure in Developing Communities
}

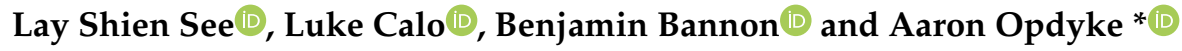 \\ School of Civil Engineering, The University of Sydney, Sydney, NSW 2006, Australia; \\ 1see4687@uni.sydney.edu.au (L.S.S.); lca19891@uni.sydney.edu.au (L.C.); bban9315@uni.sydney.edu.au (B.B.) \\ * Correspondence: aaron.opdyke@sydney.edu.au; Tel.: +61-2-8627-9049
}

Received: 31 May 2020; Accepted: 29 June 2020; Published: 1 July 2020

\begin{abstract}
Urban flooding in developing countries represents a growing threat to sustainable development efforts, yet the tools needed to study these infrastructure systems in data-scarce environments are woefully inadequate. This study seeks to propose a standardized approach and methods for mapping urban drainage systems in developing communities. The research draws on a case study from the Philippines, which sought to conduct rapid elevation surveys and drainage assessments employing open source geographical information system (GIS) tools. We develop a standardized procedure for digitizing drainage systems using OpenStreetMap and Field Papers, as well as discuss applications of this data for drainage design. The results contribute to a methodological framework that can be replicated in other similar developing communities where study of urban drainage is needed for sustainable development and disaster risk reduction efforts.
\end{abstract}

Keywords: OpenStreetMap; stormwater; flooding

\section{Introduction}

Flooding is responsible for the highest number of disaster-related fatalities of any natural hazard globally [1]. In Asia, these statistics are even more stark, with flood-related disasters estimated to account for $84 \%$ of all disaster fatalities over the last two decades [2]. These flood events are particularly devastating in developing countries with tropical climates where communities experience disproportionate flood-induced health issues, displacement, and significant loss of life [3]. It is anticipated that the frequency and magnitude of these events will be exacerbated by climate change [4]. This trend will be further intensified by anthropogenic activities such as urbanization, deforestation, and poor floodplain management. As urban sprawl grows, there is demand to monitor urban development and planning to ensure the resilience of emerging cities against the impacts of future flood events [5,6].

Urban drainage constitutes a vital part of water infrastructure systems in cities by limiting interruption of transportation services, protecting property, and inhibiting flood-related health issues [7]. Modern stormwater management has progressed to employ innovative environmentally conscious solutions to improve the quality of stormwater runoff; however, in developing countries, the main issue is still focused on managing the quantity of stormwater runoff to minimize loss of life, injury, and property damage [8]. The implementation of urban stormwater systems in developing countries is often more complex due to demanding socioeconomic, technological, and climatic conditions [9]. Developing communities are also experiencing urbanization at more rapid rates without adequate investment in infrastructure and local institutional capacity [10]. Unplanned and informal establishment of urban settlements results in the growth of impermeable surfaces and, consequently, local flooding [11,12]. Urban drainage is frequently considered as a secondary priority compared to larger infrastructure such as dams, seawalls, and reservoirs as it constitutes a less visible type of 
mitigation against flood-related hazards. As a result, poorly constructed stormwater systems persist as an issue in developing communities. These ineffective systems often fail to cope with the stormwater runoff volumes generated in growing urban landscapes during rainfall events, which exacerbates even small-scale floods.

While these issues are often known to communities who suffer from recurrent flooding, the underlying drivers are often challenging for local governments to action as data on informal and inconsistent urban drainage systems rarely exist. It is challenging for local engineers and urban planners to conceptualize designs for these stormwater systems without first having detailed information about these structures [13]. Mapping existing urban drainage infrastructure can aid in addressing these knowledge gaps and can facilitate appropriate action. Evaluation of existing drainage networks can help inform cost-effective recommendations by prioritizing locations in need of maintenance or repair. Vital characteristics of urban drainage systems, such as dimensions and construction materials, that are systemically mapped can also assist settlement-scale flood mitigation.

However, current practice struggles with the limited toolsets for basic mapping purposes in developing communities. For instance, geographic information system (GIS) data in developing countries are mainly obtained from remote sensing, which is dominated by information on land cover [14]. On-site observations are resource intensive and require coordination among various departments that often lack adequate training and equipment [15]. Although many GIS and open mapping data projects are currently emerging in developing communities $[16,17]$, there is a need for standardized practice of urban drainage mapping within resource-constrained community contexts to ensure high-quality and consistent data. How these data are collected is often neglected, yet this plays an important role in answering broader questions of how these systems perform. Such protocols have a potential for open mapping projects to be used for a wider range of technical applications, such as engineering design, in the absence of other traditional datasets.

We aim to propose a standardized approach and methods that can be used in mapping urban drainage infrastructure in developing communities where data scarcity requires distinct toolsets. After reviewing previous fieldwork mapping tools, we present a case study from a municipality in the Philippines to examine lessons from an urban drainage mapping project, and then discuss implications for advancing flood risk reduction.

\section{Background}

Geographic information systems (GISs) collect, store, process, and visualize large volumes of geographical data to understand spatial relationships. Besides mapping capabilities, GIS links spatial and attribute data to define information about features [18]. The coupling of these two data enables spatial analysis and visualization that are vital for making evidence-based decisions in the fields of engineering and development. GIS technology plays a vital role in flood-related disaster risk reduction measures such as flood simulation and modeling, floodplain management, and the development of early warning systems $[19,20]$. Mapping infrastructure, such as roadways and buildings, contributes to developing flood risk assessments and emergency route plans to minimize loss of lives. During disaster response and relief, it is also commonly used as a key tool for humanitarian agencies and first responders to quickly assess emergency situations and coordinate response efforts [21].

GISs can provide an understanding of landscapes and features to support decision-making processes during urban planning and flood mitigation [22]. It is crucial in traffic, environment, recreation, land use, and social service planning, as well as in urban stormwater management. However, in developing countries where urban flooding remains a complex issue, urban planners and engineers struggle with the lack of formal spatial data on existing stormwater systems [23]. The mapping applications of GIS technology have potential to bridge this gap by allowing the visualization of existing urban drainage infrastructure, even in resource-constrained settings. Key information on existing drainage networks can be integrated to provide a more comprehensive understanding of current systems. 
While there are many advanced GIS technologies available for mapping applications, mapping approaches in developing countries are often met with significant constraints. Foremost, local governments rarely have digital versions of maps essential for planning. The quality of available data in rural regions also tends to be questionable as there is generally insufficient formal coordination among different departments in updating and monitoring geo-datasets. The lack of financial resources, expertise, and availability of GIS software in developing communities also creates a challenging platform for mappers to collect and process data.

Therefore, the question arises-how can toolsets be made more available to local governments with limited formal GIS training and how can they be used to understand unsystematic drainage networks in urban settings? In developing communities, the challenges often have less to do with the availability of GIS methods, as there are a growing number of open source options, than with the ability to utilize these technologies to advance flood risk reduction. While remote sensing technologies have produced significant advances in understanding both the built and natural environments in developing countries where data are sparse, the available resolution of these data often still necessitates field efforts to collect supplementary technical information.

These challenges call for distinct toolsets for the implementation of mapping projects in developing communities. There is a need to involve local organizations and engage with communities throughout project planning and execution [24,25]. Training and capacity building of local expertise is vital for the co-creation of maps and datasets, including protocols of mapping procedures involving methods of data collection, digitization, and processing. Mapping projects in these contexts need to incorporate equipment and GIS software with minimal maintenance costs to ensure the sustainability of these mapping practices.

Open data has growing potential to allow for collaborative mapping and public participation to overcome the lack of geo-information data in resource-constrained settings [26,27]. OpenStreetMap (OSM) is a collaborative mapping tool central to the goal of allowing an openly editable global map to the public. OSM is utilized for humanitarian response, disaster prevention and management, and urban planning. It is increasingly a tool for local governments, humanitarian actors, and development agencies. The technology has been applied in many developing countries to advance resilient local development. However, despite novel applications, there are relatively few instances where OSM has been applied to understanding urban drainage systems.

Gebremedhin et al. [28] mobilized participatory approaches in developing improved urban flood models in Tanzania. Using OSM as a shared platform, they developed 1D-2D coupled flood models in Dar es Salaam through open mapping approaches, demonstrating the value of well-defined participatory processes in data-scarce regions. Their results also demonstrated the potential of open data in developing flood models for further hydraulic analysis of inundation through waterways and floodplains. Crowdsourcing mapping methods are especially effective under conditions where there is limited historical data, such as urban flooding, and community perceptions can be used as a means to fill gaps in traditional datasets. For example, Klonner et al. [29,30] applied this concept in combining quantitative OSM and qualitative community spatial data in Santiago, Chile. The generated maps highlighted sensitive areas of interest for flood management. Other participatory approaches to mapping drainage for flood risk reduction have also continued to emerge [31].

While there are a growing number of urban drainage mapping approaches in various flood management initiatives, there is a gap in the standardized practice for capturing informal drainage networks using OSM in urban settlements with limited resources. With a growing focus on urban stormwater management as a fundamental component of disaster risk reduction, we build on the experience of previous mapping projects in developing communities to propose a standardized approach in mapping urban drainage in developing regions. This approach will allow future researchers and local governments with limited resources to better understand the present drainage infrastructure and facilitate urban flood management. 


\section{Methods}

We draw on case study methods to present a collaborative urban drainage mapping project from the Philippines. We will discuss the mapping protocol that emerged from this work while engaging closely with local actors to co-create a process of urban drainage mapping, as well as the analytical procedures that were used to generate final urban drainage maps. We propose a mapping protocol, shown in Figure 1, to provide a standardized approach for urban drainage mapping projects in developing settings.

This research was conducted in close collaboration with the Municipality of Carigara, located in the province of Leyte in the Philippines. Carigara is a second-class municipality, as defined by the Philippines government, and covers approximately $120 \mathrm{~km}^{2}$ in its geographical extent. It is home to a population of more than 50,000 residents. During rainy seasons, flooding is commonly observed, causing social and economic disruption. While there are a number of factors impacting the nature of flood hazards in Carigara, such as climate change and illegal logging activities, infrastructure vulnerability also plays a role. There are many discontinuities in the existing drainage networks and stormwater flows regularly exceed the volumetric capacity of drainage networks. However, while drainage was identified as a pathway to risk reduction, much of this knowledge was held within communities and not formalized in municipal planning efforts. Furthermore, specific households could often point to localized areas where drainage improvements were needed, but the local government noted the difficulty in formulating an overall stormwater management strategy.

Our fieldwork was focused within the urban, coastal areas in nine barangays, the lowest political division in the Philippines. These high flood risk communities included Ponong, Sawang, Baybay, Jugaban, San Mateo, Guindapunan West, and Visoria East and West. We divided drainage mapping into two components: (1) an elevation survey and (2) drainage assessments. These aimed to map the location of drainage networks as well as other characteristics including dimension, slope, condition, and surface lining material. The assessments were completed in collaboration with local community members and Carigara's Disaster Risk Reduction and Management Office (DRRMO).

\subsection{Defining an Assessment Boundary}

Before commencing our fieldwork and mapping processes, we engaged with the local DRRMO staff and barangays' representatives to identify common flood zones throughout the urban coastal regions. Local resources and capacities available were also identified to establish fieldwork teams. One survey team and two drainage assessment teams were assigned to each barangay. The survey team consisted of two surveyors with the help of traffic control by a few barangay tanods (community police). Each drainage assessment team was led by a mapper and at least two personnel to assist in measuring drainage dimensions.

\subsection{Pilot Observation and Mapping}

We selected a preliminary observation area to conduct a pilot observation to first understand the local urban stormwater system. During the pilot observation, we carried out an elevation survey around several building blocks. The process of our elevation survey is detailed in the following section. Next, we performed a preliminary drainage assessment to identify different types of drainage characteristics to be documented, as presented in Table 1. A protocol on different classifications for drainage conditions and surface material was developed through pilot observations and engagement with local personnel and was supplied to all data collection teams with a description and example photos. 
Step 1: Defining an assessment boundary

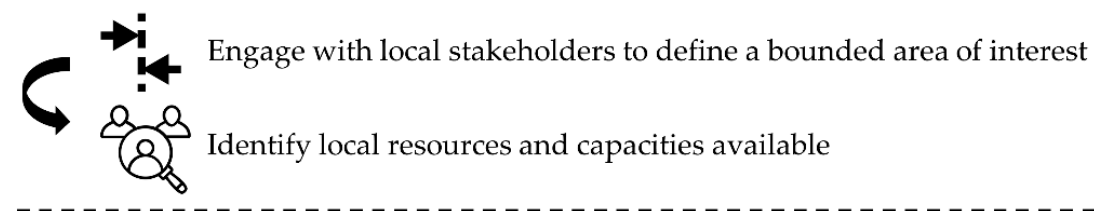

\section{Step 2: Pilot observation and mapping}

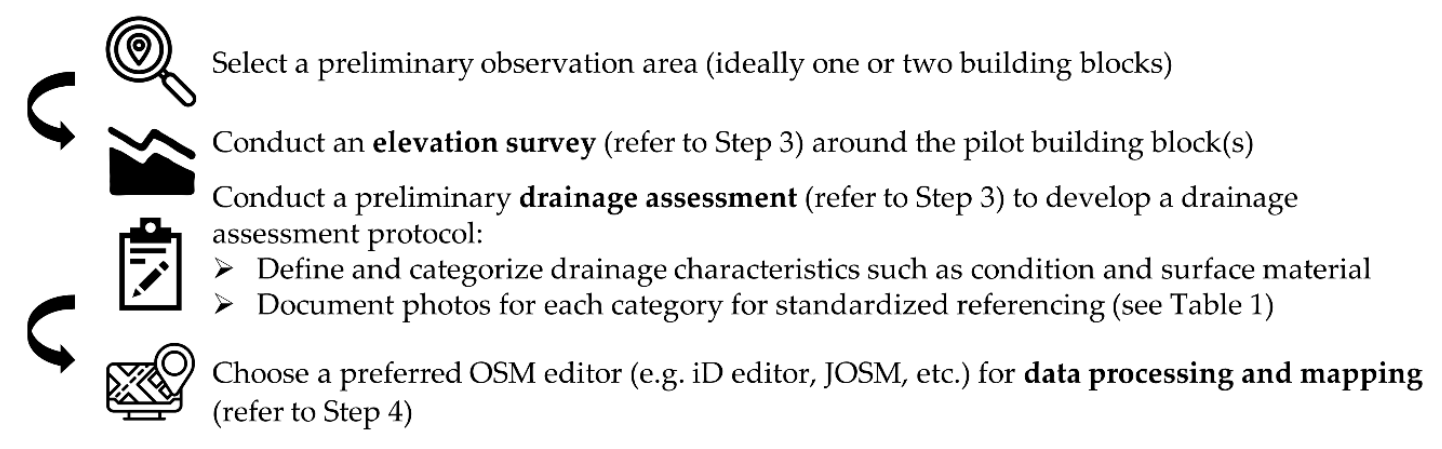

\section{Step 3: Data collection}

\section{Elevation survey}

* Define survey runs (limit run length to an appropriate distance depending on the survey method and equipment used)

(1) Collect elevation points at all road intersections

(2) Take additional elevation measurements along main waterways (rivers/streams)

(3) Measure invert elevations at bridge crossings

(4) Record elevation values on Field Papers

\section{Drainage assessment}

(5) Trace location of the drainage along every street and note the drainage flow direction on Field Papers

(6) Measure and record the cross-section dimensions at the ends of each street block (when intersecting drains are of different sizes, measure the cross-section at the entering stretch of each channel)

(7) Record the average condition of drainage (e.g. good, fair or poor)

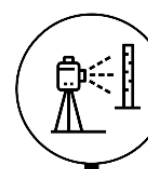

\section{ans}

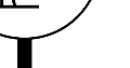

.

Note material of base surface (e.g. concrete or earth)

\section{Step 4: Data processing and mapping}

Correct elevations to local datum (if available) and digitize point elevations (at roadway intersections or points assigned to ways)

Scan Field Papers notes and digitize drainage data

$>$ Assign dimensions, condition and surface as keys and tag corresponding values

$>$ Calculate and assign the drainage slope (based on drainage depth and nearest elevation)

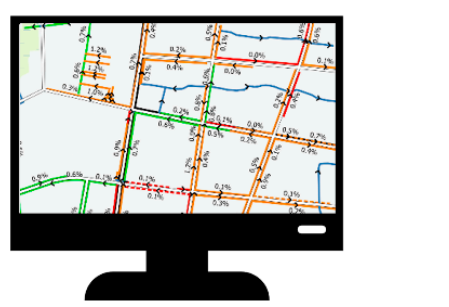

Import OSM data into QGIS (or other GIS software) for styling and visualization

Figure 1. Open data mapping process. OSM: OpenStreetMap; JOSM: Java OpenStreetMap; GIS: geographic information system; QGIS: Quantum Geographic Information System. 
Table 1. OSM drainage tagging classifications.

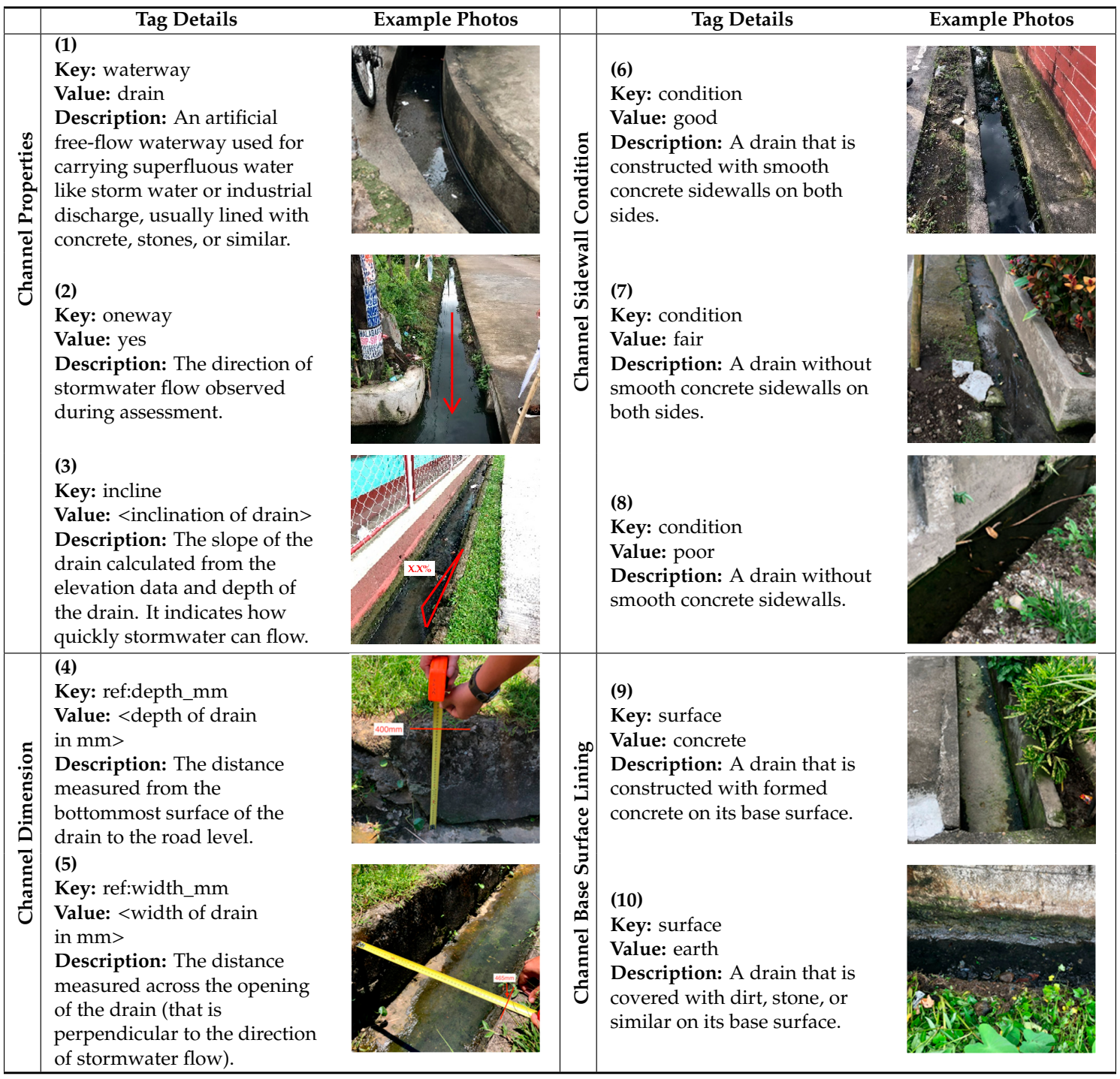

A preliminary mapping process was also carried out using JOSM (Java OpenStreetMap) to record elevation values and digitize drainage channels documented from field observations. During this process, training workshops were conducted to familiarize local personnel with mapping techniques and standard OSM processes.

\subsection{Data Collection}

We divided our data collection into two parts that included an elevation survey and drainage assessments, which were then combined in our analysis, which will be discussed below.

\subsubsection{Elevation Survey}

An elevation survey was conducted to determine a ground surface for the areas of interest. This was completed by collecting elevation points at all road intersections throughout the considered nine barangays. While previous ground surveys and remote sensing data existed, none of these were of sufficient resolution, or recent, to afford capturing elevations of the in situ drainage network. The survey was carried out using an automatic level and leveling staff. While the manual survey process was a more time-intensive process compared to other alternatives (vs. Global Navigation Satellite System (GNSS)-based survey methods), it was significantly cheaper and required lower 
long-term maintenance of equipment. This was ideal for the local government to build upon local capacities to carry out similar surveys in the future.

Survey runs were subdivided within barangay boundaries to limit run lengths to $100 \mathrm{~m}$. A total of 260 elevation points were collected. Additional elevation measurements were taken along main waterways (e.g., streams) in order to increase the resolution of the digital elevation model (DEM). Invert elevations were also measured at all bridge and culvert crossings. All elevation points collected were referenced to the World Geodetic System (WGS) 84 datum and benchmark LY-149 created in 2007 by the Philippines' National Mapping and Resource Information Authority (NAMRIA). Results of the elevation survey were then used to develop a DEM of the ground terrain of the municipality to help understand flow paths.

\subsubsection{Drainage Assessments}

The drainage assessment was conducted by observations along every street in the urban areas of coverage. The aim of the survey was to record drainage characteristics such as the location, dimensions, slope, sidewall condition, and material of base surface lining. We chose to collect data on these characteristics as they are the basic qualities of a drainage channel and aligned with expected future maintenance work. Field Papers (http://fieldpapers.org/), a free, automated georeferenced atlas tool, was used extensively during the fieldwork to generate field maps to record these on-site observations.

We recorded the exact location of the drainage and the direction of stormwater flow on Field Papers according to our observations during the survey. To record the sizing of drainage, we measured the cross-section dimensions at the ends of each street block. These measurements were recorded in millimeters for the depth from the bottommost surface of the drain to the road level and the width was measured perpendicular to the direction of stormwater flow. In cases where the intersecting drains were of different sizes, measurements were recorded for each and assigned to the entering stretch of drainage. To obtain the slope of the drainage channel, the depth recorded was later combined with center-of-road elevations.

We categorized and recorded the condition of drainage as good, fair, or poor. Good condition was defined as drainage that was constructed with smooth concrete sidewalls with no signs of damage, while drainage with unlined sidewalls were considered under poor condition. Drainage that was lined with only one concrete sidewall was assigned a fair condition. These three main classifications represented the condition of the sidewalls, which was deemed to be the one of the most important factors for stormwater transport, while keeping the survey recording simple. Furthermore, the material of the base surface was noted as either concrete or earth. Well-constructed drainage was commonly lined with smooth concrete while a drain with dirt, stone, or similar base surface was recorded as earth.

\subsection{Data Processing and Mapping}

OpenStreetMap was used to plot elevation points, map drainage channels, and digitize field notes. We used JOSM (Java OpenStreetMap), which is an extension editor of OSM, equipped with more advanced tools. The ability of JOSM to function offline also makes it useful in regions with limited internet connectivity, as was our context. Quantum Geographic Information System (QGIS) software was also used in parallel with OSM in the processing and visualization of data obtained.

Elevation data were added to road intersection nodes using JOSM. These elevation values were tagged under the key "ref:elev." The data were then imported into QGIS with the QuickOSM plugin. We created a DEM from the discrete elevation points through triangulated irregular network (TIN) interpolation methods. The TIN method helps to create a continuous terrain surface by connecting irregularly located points to adjacent points to form non-overlapping and compacted triangles with elevation values stored at the nodes [32]. This method was chosen as it can yield the most accurate results from sample points that were scattered unevenly from the measurements at every road intersection.

Similarly, we digitized the collected on-site drainage data using JOSM. We plotted drainage as ways in JOSM and tagged with the "waterway" key and "drain" as its corresponding value based 
on the tagging conventions used in the Philippines [33]. The stormwater flow directions were also assigned following field observations. We referred to the flow direction of nearby drainage for areas where the channels were dry during assessment and there was no information on the direction of flow. The ways were then tagged with "oneway" as the key and "yes" as its corresponding value; this step was added for visualization of drainage maps later. As dimensions of drainage were measured at discrete locations along the drainage channel, a method of averaging was applied to assign a simplified dimension along the entire length. The averaged dimensions were tagged as "ref:depth_mm" and "ref:width_mm." The term "ref:" was included to indicate a local tag while the unit "mm" was included since the conventional unit of measurement used in OSM is commonly in meters. We propose this as a possible standard tag when tagging future drainage infrastructure in OSM, which should be validated in consultation with local mappers. The slope of the drainage was tagged as "incline" in OSM, calculated from the drainage depth and surveyed elevation values, as

$$
\text { Slope }(\%)=\frac{\left(\text { Elevation }_{\mathrm{A}}-\text { Depth }_{\mathrm{A}}\right)-\left(\text { Elevation }_{\mathrm{B}}-\text { Depth }_{\mathrm{B}}\right)}{\text { Distance between A and B }} \times 100 \%
$$

where $\mathrm{A}$ and $\mathrm{B}$ are two opposing ends of a drainage channel. Figure 2 shows a basic drainage flow model in which the solid red line represents the bottom surface of the drainage. The base slope of the channel is not necessarily parallel to the slope of the road surface; it depends on the change of depth along the drainage and is the key factor in determining the direction of stormwater flow. During our fieldwork, different teams were delegated to conduct the elevation survey in parallel with the drainage assessment to speed up the data collection process. The results of the survey and assessment were later combined using JOSM to calculate the slope values during data processing. Slope values are expressed as percentages and were tagged under the key "incline".

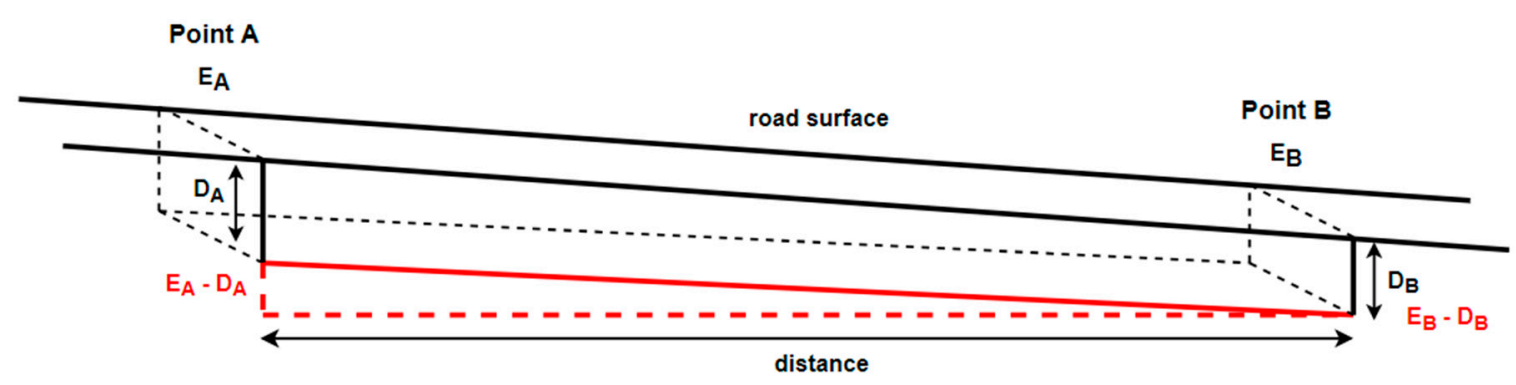

Figure 2. Drainage flow model.

\section{Results}

The mapping process resulted in an elevation and drainage map, shown in Figure 3. We used color ramps to visualize the DEM of the barangays with red being the higher elevations and blue denoting lower regions. We also added contour lines of a $0.5 \mathrm{~m}$ resolution to highlight areas with rapid change of slope. These maps sought to inform future drainage design by providing information on the natural contours of central urban areas and nearby waterways. 


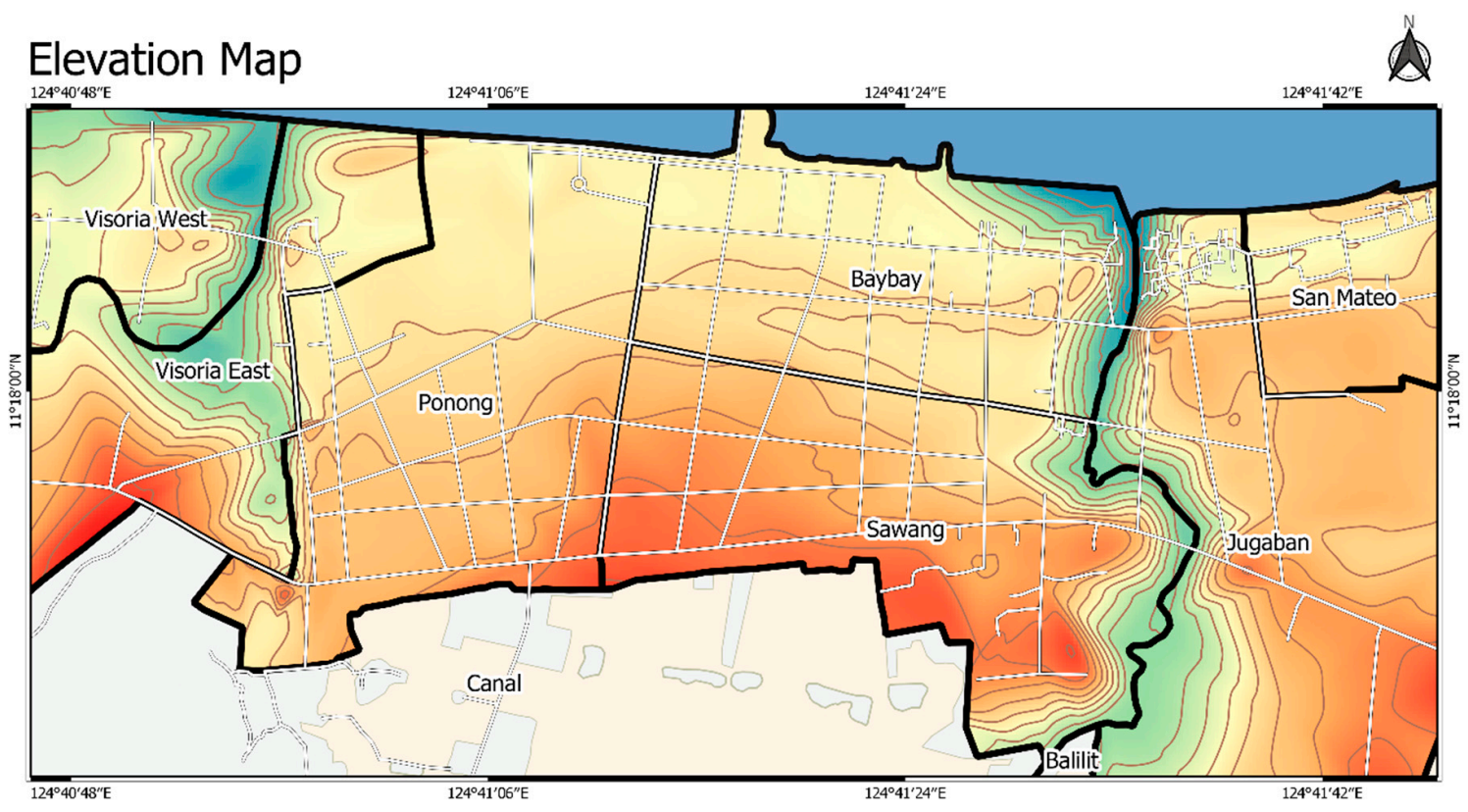

Drainage Map

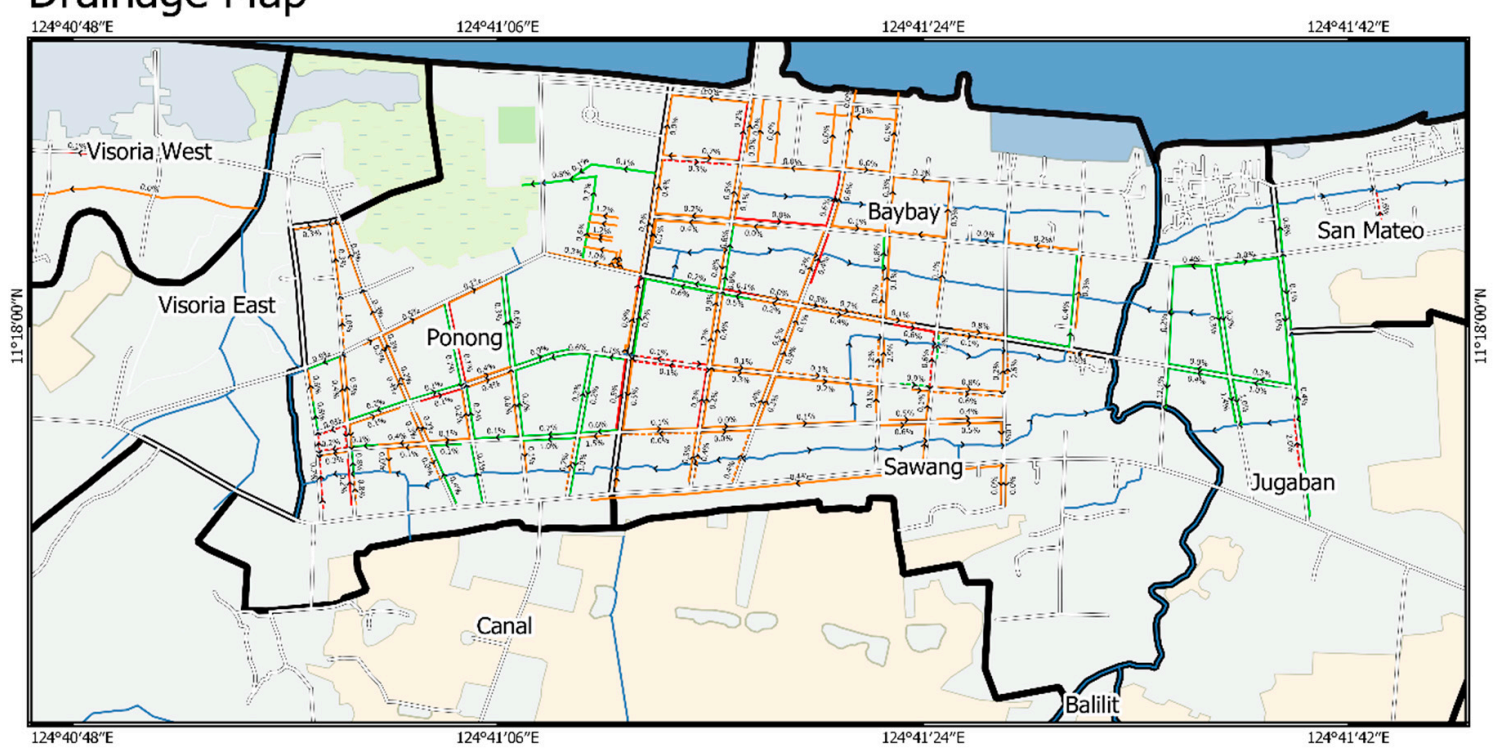

\section{LEGEND}

Elevation (m)
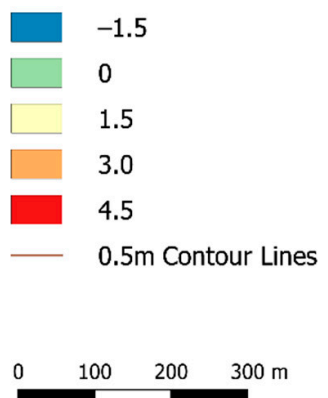

Drainage

_ Good condition lined with concrete Good condition lined with earth

- Fair condition lined with concrete Fair condition lined with earth Poor condition lined with concrete Poor condition lined with earth $>$ Drainage flow direction

X.X\% Drainage slope

- Municipal canals

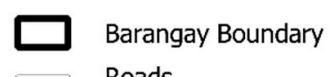
Roads

Land Cover

Residential

Farmland

Scrub

Mangrove

Water

Ocean

Figure 3. Elevation and drainage maps.

The drainage map incorporated characteristics we recorded during drainage assessments. Drainage channels were mapped with green, orange, and red to represent good, fair, and poor 
conditions, respectively. Solid lines indicate smoothly lined concrete channels while dotted lines reflect channels with bare earth linings. The arrows specify the drainage flow direction while the percentage values denote the channel slopes. The dimensions of the channels were not presented on this map to avoid overcrowding of labels but were included as an attribute of the GIS layer which can be exported to create channels in flood models for future hydraulic studies. The municipal canals (represented by blue lines) were channels that existed in OSM from previous mapping projects conducted in Carigara. The drainage map highlights drainage issue hotspots that can inform effective stormwater maintenance and locations in need of reconstruction or repair. In total, the assessments mapped 19,925 m of drainage in a two-week period.

Observations from the drainage assessment highlighted three primary issues that were observed to impact the effectiveness of the system in transporting stormwater. We will present these below, in order of importance, along with recommendations. While we will discuss the specific system studied, our results are beneficial to other local governments in the Philippines whose systems are similar.

\subsection{Connectivity and Slope}

Results from the drainage survey revealed that many channels were poorly connected. The discontinuities throughout the drainage network revealed the root cause of stagnation issues that were commonly observed during heavy rains. Many channels were also constructed with insufficient slope, leading to accumulation of stormwater in the system that caused ponding under small rainfall events. Our analysis of slopes also found that in some cases, channels on opposite sides of the same road transported stormwater in opposite directions. While there is no inherent problem if sufficient flow paths are available, this adds to the complexity of the system and complicates long-term maintenance where field crews have difficulty determining flow directions. These issues in connectivity and slope stem from the ad hoc basis in which channels were added in the municipality, which led to disjointed connections. Future drainage investments should focus on improving the links between existing drainage to allow stormwater to flow effectively throughout the drainage network and safely discharge downstream. Besides connecting adjacent drainage, the system should also encourage stormwater discharge to the larger municipal canal and river systems to enhance outflow from the urban center to decrease community flood risk. These larger channels were observed to be underutilized despite being well sized and of high-quality construction.

\subsection{Construction Quality}

We found that $27 \%$ of drainage channels were in good condition, $62 \%$ were in fair condition, and $10 \%$ were in poor condition, based on length. Concrete lining was present in $86 \%$ of channels with the remaining $14 \%$ being earth lined. Although many channels were lined with concrete as the base surface, most of the channels still had earthen sidewalls. In hydraulic analysis, this contributes to a higher Manning's value based on the cumulative rough surface areas, which eventually leads to less effective stormwater transport. These undeveloped surfaces also more easily allow vegetation growth, which disrupts stormwater flows. To minimize surface roughness and irregularities, unlined drainage channels should be replaced with concrete material throughout the wetted perimeter of the channel. General construction improvements were observed to be needed to increase channel uniformity.

\subsection{Maintenance}

It was common to observe vegetation or rubbish trapped in drainage, which communities noted frequently led to flooding and pollution issues. The combined used of drainage for domestic wastewater also was observed to stress the system. It was especially evident in areas near the central market, where waste was frequently disposed into channels, adding high contaminant loads. Solving the underlying causes of these issues requires long-term behavior change and more robust policy that is enforced [8], however preventative maintenance involving the removal of vegetation and rubbish during regular cleaning of drainage would also greatly enhance observed blockages. Communities 
noted that while these there was regular maintenance conducted in central areas and in areas where blockages caused flooding, outlying channels that are vital in large rainfall events should receive greater attention. With regular maintenance work, the stormwater system can perform better and also reduce exposure of the community to health issues.

\section{Discussion}

The pilot observations conducted were an important step in understanding the local urban stormwater systems, for both the local and international team members, and these provided valuable insights in the development of a robust drainage assessment protocol for site-specific conditions. Engagement with local officials and communities during the pilot observation was important for mappers to identify flood-prone areas, notably near critical infrastructure such as schools or markets.

When developing the atlas through Field Papers, it was important to select a suitable scale that provided sufficient paper space to make notes on pages without overcrowding the printed atlas affecting the digitization process. While defining a drainage assessment protocol, it is important that end-users be involved in the decision-making processes to discuss additional drainage characteristics and how the data will be used in future local development efforts. For example, identifying blocked or damaged drainage was of particular interest to the local government, in this case, the municipality. This information was desired for its benefit in planned maintenance work.

Furthermore, tagging classifications should adhere to existing OSM guidelines, if available. At a minimum, consistent tagging conventions and measurement units should be defined for all mapping teams. Documentation of photos during our pilot observation was key in defining a standardized description for different characteristics and ensuring a consistent categorization. The tagging protocol developed in Table 1 may be useful for future drainage mapping efforts. Training workshops were included as part of our process and the time investments for these should be accounted for in upfront planning to familiarize local personnel with mapping techniques and standard OSM processes. This step is crucial in ensuring a common understanding of all processes from data collection to digitization. The pilot mapping process can also incorporate mapping other elements such as buildings and roads in cases where there is limited existing OSM data, as this may be important to orient assessment teams.

Once a clear protocol is defined, different teams should be delegated to carry out elevation surveys and drainage assessments in parallel. One survey team and two drainage assessment teams were assigned to each barangay during our fieldwork. With the number of fieldwork staff available, approximately three hours were required to complete the elevation survey and drainage assessments of each of the barangays, which averaged around 2.4 hectares. The boundary of the focus area should be clearly defined to prevent clashing teams during data digitization. Communication with local communities who have extensive knowledge is also critical during drainage assessments to provide a comprehensive understanding of the systems. During our fieldwork, we used a checklist of questions:

- In which direction does the water flow along this drainage?

- Is there a culvert located under this road intersection?

- Is there a channel passing through under your settlement?

- Does ponding of rainwater occur here?

- Does drainage blockage often happen near your settlement?

These questions can be modified in other communities according to the local context and different types of drainage. One of the main hurdles in improving urban stormwater systems in developing communities is the lack of community input as drainage solutions are often designed without the involvement of affected households [8]. The aim of the above questions is to assist in mobilizing local knowledge to aid in creating effective investment in a future stormwater management strategy.

The last step of the process should involve digitizing field data in OSM editing software and processing data to produce desired outputs. We consciously mapped the drainage channels of a barangay immediately after the area was surveyed and assessed during fieldwork. This allowed 
mappers to carry out quick data checks to identify gaps or errors in mapping in case a re-assessment was required before moving on to the next community.

While there are numerous methods of managing sustainable drainage, few tools are tailored to the type of informal systems found in developing communities. The use of OSM can be a powerful platform for local governments to capture monitoring data on these networks. As we have shown, collecting and mapping basic channel characteristics builds a foundation for more detailed future analysis of these small-scale drainage systems. Beyond the immediate technical lessons learned, the project also afforded insights on how to mobilize knowledge for disaster risk reduction. Many of the local staff involved reflected on the ability of the systematic approach to make visible problems they passed every day. The condition maps in particular revealed larger regions where risk is accumulated. In many cases, this risk is formed as a result of additive decisions where infrastructure systems are often built incrementally in developing communities. Understanding settlement-level risk requires taking stock of overall systems - their connections, characteristics, and boundaries. This case study also shows the importance of considering smaller drainage infrastructure in disaster risk reduction strategies.

\subsection{Limitations}

We outline a few limitations of the mapping protocol to provide context on adaptations for the implementation of future urban drainage mapping projects. While we believe the fieldwork and mapping approach proposed is generalizable across similar developing communities, the methods were developed and improvised based on the study of one municipality in the Philippines. Therefore, we encourage these approaches to be adapted for different national contexts other than the Philippines where types of drainage and local conditions may differ. OSM tagging conventions may vary in different contexts and should adhere to local keys and values documented in available OSM wiki pages to allow for consistent data interpretation.

Further, the practicality of our protocol was developed for open, gravity drainage systems. The mapping of underground pipes would be significantly more challenging using the applied techniques. Our framework was also developed for combined stormwater and sewer networks. The mapping of separate systems in communities may require further tags and protocols to ensure these are adequately assessed and digitized. Consulting local personnel during the pilot observations should take place to ensure the attributes are applicable for future local development works. Our protocol should be adapted to meet project-specific needs; however, our work outlines a standardized approach for mapping urban drainage in data-scarce developing regions. With proper institutional arrangements, local support, technical skills, and resources, developing communities can benefit from the resulting open data that can be applied to improving urban stormwater management [23].

\subsection{Future Research}

With many developing communities lacking the tools and data to address complex urban challenges, we believe future research should continue to promote the use of open data and participatory approaches in urban flood mitigation. While this study focused on mapping urban drainage infrastructure, future mapping projects, whether initiated by international organizations or local communities, should widen the scope of mapping to incorporate other infrastructure that impacts urban water systems such as land cover, roads, and buildings. There is also a need to look more closely at the open data that already exist and their potential role in design. For example, Schilling and Tränckner [34] developed a method of estimating wastewater volumes with high spatial resolution based on OSM building data. Studies such as these are needed to understand the usability of volunteered geospatial infrastructure data in the design and assessment of stormwater and wastewater systems. There are many emerging GIS-based technologies and approaches aimed at filling gaps of missing data and generating realistic flood models at a city scale in data-scarce areas. The development of these models can contribute to hydraulic studies and promote an improved understanding of local flooding behavior. 


\section{Conclusions}

Drawing on a participatory action research in collaboration with a local government in the Philippines, we presented a case study of an urban drainage mapping project. Through this process, we developed a protocol for the mapping and assessment of open channel, gravity drainage systems in developing communities using OpenStreetMap. This approach outlines key variables for urban flood modeling and proposes standardized open data tagging of dimensions, conditions, and base surface materials - key variables in the design and maintenance of drainage systems. Our work contributes to defining clear data collection protocols for the capture of informal urban drainage networks.

Our partnership approach also presents a framework for future capacity building of local engineering, surveying, and disaster risk reduction staff. While we assisted in conducting the surveys and assessment, the maps were co-created by members of the local community, which created a sense of local ownership in the outputs. The target local government will be able to build on results from the elevation survey and drainage assessment for future urban stormwater works and planning strategies.

Many developing communities face issues of data scarcity, struggling to plan for rapid urbanization. By presenting a standardized protocol for mapping urban drainage in data-scarce regions, this study allows future researchers to employ simple approaches to bridge the knowledge gap of current drainage infrastructure in developing communities. Having utilized OSM, the methodology we adopted is easily replicated in other similar developing community contexts where data scarcity hampers urban flood management [34]. Adopting a participatory action approach also adds to local capacity in flood management, leveraging the use of GIS tools to facilitate community ownership and voice. This case study demonstrates the importance of a clear mapping framework to guide drainage assessments. Moreover, active involvement of the local community in the process of data collection, processing, and mapping plays a vital role in organizing the local knowledge of these systems. By addressing data gaps of informal urban drainage structures, local decision-makers can acquire the information they need to advance future urban drainage design and disaster risk reduction strategies.

Author Contributions: Conceptualization, A.O.; formal analysis, L.S.S., L.C., and B.B.; funding acquisition, A.O.; investigation, L.S.S., L.C., and B.B.; methodology, L.S.S., L.C., and B.B.; resources, A.O.; software, L.S.S.; supervision, A.O.; validation, L.S.S.; visualization, L.S.S.; writing—original draft, L.S.S.; writing—review and editing, A.O. All authors have read and agreed to the published version of the manuscript.

Funding: This research was supported by an Early Career Researcher Development Grant from the Faculty of Engineering at the University of Sydney.

Acknowledgments: We wish to thank the Carigara Municipal Disaster Risk Reduction and Management Office for their assistance in collecting the data and for their support.

Conflicts of Interest: The authors declare no conflict of interest.

\section{References}

1. Doocy, S.; Daniels, A.; Murray, S.; Kirsch, T.D. The Human Impact of Floods: A Historical Review of Events 1980-2009 and Systematic Literature Review. PLoS Curr. Disasters 2013. [CrossRef] [PubMed]

2. Guo, H. Understanding global natural disasters and the role of earth observation. Int. J. Digit. Earth 2010, 3, 221-230. [CrossRef]

3. Ohl, C.A.; Tapsell, S. Flooding and human health. BMJ 2000, 321, 1167-1168. [CrossRef]

4. Hirabayashi, Y.; Mahendran, R.; Koirala, S.; Konoshima, L.; Yamazaki, D.; Watanabe, S.; Kim, H.; Kanae, S. Global flood risk under climate change. Nat. Clim. Chang. 2013, 3, 816-821. [CrossRef]

5. Few, R. Flooding, vulnerability and coping strategies: Local responses to a global threat. Prog. Dev. Stud. 2003, 3, 43-58. [CrossRef]

6. Zhou, Q.; Leng, G.; Su, J.; Ren, Y. Comparison of urbanization and climate change impacts on urban flood volumes: Importance of urban planning and drainage adaptation. Sci. Total Environ. 2019, 658, 24-33. [CrossRef] [PubMed]

7. Fryd, O.; Dam, T.; Jensen, M.B. A planning framework for sustainable urban drainage systems. Water Policy 2012, 14, 865-886. [CrossRef] 
8. Silveira, A.L.L. Problems of modern urban drainage in developing countries. Water Sci. Technol. 2002, 45, 31-40. [CrossRef]

9. Parkinson, J.; Tayler, K.; Mark, O. Planning and design of urban drainage systems in informal settlements in developing countries. Urban Water J. 2007, 4, 137-149. [CrossRef]

10. Cohen, B. Urbanization in developing countries: Current trends, future projections, and key challenges for sustainability. Technol. Soc. 2006, 28, 63-80. [CrossRef]

11. Gupta, K. The drainage systems of India's cities. Waterlines 2005, 23, 22-24. [CrossRef]

12. Abd-Elhamid, H.F.; Zele, M.; Vranayova, Z.; Fatyh, I. Evaluating the Impact of Urban Growth on the Design of Storm Water Drainage Systems. Water 2020, 12, 1572. [CrossRef]

13. Parkinson, J. Urban drainage in developing countries-Challenges and opportunities. Waterlines 2002, 20, 2-5. [CrossRef]

14. Yeh, A.G.-O. The development and applications of geographic information systems for urban and regional planning in the developing countries. Int. J. Geogr. Inf. Syst. 1991, 5, 5-27. [CrossRef]

15. Câmara, G.; Fonseca, F. Information policies and open source software in developing countries. J. Am. Soc. Inf. Sci. Technol. 2007, 58, 121-132. [CrossRef]

16. Missing Maps. Available online: https://www.missingmaps.org/ (accessed on 31 May 2020).

17. Scholz, S.; Knight, P.; Eckle, M.; Marx, S.; Zipf, A. Volunteered Geographic Information for Disaster Risk Reduction-The Missing Maps Approach and Its Potential within the Red Cross and Red Crescent Movement. Remote Sens. 2018, 10, 1239. [CrossRef]

18. Tian, B. GIS Technology Applications in Environmental and Earth Sciences; CRC Press: Boca Raton, FL, USA, 2016; ISBN 978-1-4987-7605-9.

19. McCallum, I.; Liu, W.; See, L.; Mechler, R.; Keating, A.; Hochrainer-Stigler, S.; Mochizuki, J.; Fritz, S.; Dugar, S.; Arestegui, M.; et al. Technologies to Support Community Flood Disaster Risk Reduction. Int. J. Disaster Risk Sci. 2016, 7, 198-204. [CrossRef]

20. Ouma, Y.; Tateishi, R. Urban Flood Vulnerability and Risk Mapping Using Integrated Multi-Parametric AHP and GIS: Methodological Overview and Case Study Assessment. Water 2014, 6, 1515-1545. [CrossRef]

21. Kawasaki, A.; Berman, M.L.; Guan, W. The growing role of web-based geospatial technology in disaster response and support. Disasters 2013, 37, 201-221. [CrossRef] [PubMed]

22. Eini, M.; Kaboli, H.S.; Rashidian, M.; Hedayat, H. Hazard and vulnerability in urban flood risk mapping: Machine learning techniques and considering the role of urban districts. Int. J. Disaster Risk Reduct. 2020, 101687. [CrossRef]

23. Parkinson, J. Drainage and stormwater management strategies for low-income urban communities. Environ. Urban. 2003, 15, 115-126. [CrossRef]

24. Chambers, R. Participatory Mapping and Geographic Information Systems: Whose Map? Who is Empowered and Who Disempowered? Who Gains and Who Loses? Electron. J. Inf. Syst. Dev. Ctries. 2006, 25, 1-11. [CrossRef]

25. Usón, T.J.; Klonner, C.; Höfle, B. Using participatory geographic approaches for urban flood risk in Santiago de Chile: Insights from a governance analysis. Environ. Sci. Policy 2016, 66, 62-72. [CrossRef]

26. Buchecker, M.; Menzel, S.; Home, R. How much does participatory flood management contribute to stakeholders' social capacity building? Empirical findings based on a triangulation of three evaluation approaches. Nat. Hazards Earth Syst. Sci. 2013, 13, 1427-1444. [CrossRef]

27. Kankanamge, N.; Yigitcanlar, T.; Goonetilleke, A.; Kamruzzaman, M. Can volunteer crowdsourcing reduce disaster risk? A systematic review of the literature. Int. J. Disaster Risk Reduct. 2019, 35, 101097. [CrossRef]

28. Gebremedhin, E.T.; Basco-Carrera, L.; Jonoski, A.; Iliffe, M.; Winsemius, H. Crowdsourcing and interactive modelling for urban flood management. J. Flood Risk Manag. 2020. [CrossRef]

29. Klonner, C.; Marx, S.; Usón, T.; Höfle, B. Risk Awareness Maps of Urban Flooding via OSM Field Papers-Case Study Santiago de Chile. In Proceedings of the ISCRAM 2016 Conference, Rio de Janeiro, Brasil, 22-25 May 2016.

30. Klonner, C.; Usón, T.J.; Marx, S.; Mocnik, F.-B.; Höfle, B. Capturing Flood Risk Perception via Sketch Maps. ISPRS Int. J. Geo-Inf. 2018, 7, 359. [CrossRef]

31. Zehra, D.; Mbatha, S.; Campos, L.C.; Queface, A.; Beleza, A.; Cavoli, C.; Achuthan, K.; Parikh, P. Rapid flood risk assessment of informal urban settlements in Maputo, Mozambique: The case of Maxaquene A. Int. J. Disaster Risk Reduct. 2019, 40, 101270. [CrossRef] 
32. Liang, S.; Li, X.; Wang, J. Geometric Processing and Positioning Techniques. In Advanced Remote Sensing; Elsevier: Oxford, UK, 2012; pp. 33-74. ISBN 978-0-12-385954-9.

33. OpenStreetMap Contributors Philippines/Mapping Conventions. Available online: https://wiki. openstreetmap.org/wiki/Philippines/Mapping_conventions (accessed on 31 May 2020).

34. Schilling, J.; Tränckner, J. Estimation of Wastewater Discharges by Means of OpenStreetMap Data. Water 2020, 12, 628. [CrossRef]

(C) 2020 by the authors. Licensee MDPI, Basel, Switzerland. This article is an open access article distributed under the terms and conditions of the Creative Commons Attribution (CC BY) license (http://creativecommons.org/licenses/by/4.0/). 